\title{
Domino Knoevenagel/Michael addition/C-acylation reactions. Synthesis of 1,3-cyclohexane-1,3-diones
}

\author{
Slawomir Westerlich and Tadeusz S. Jagodziński* \\ Institute of Chemistry and Environmental Protection, West Pomeranian University of \\ Technology, Aleja Piastow 42, 71-065 Szczecin, Poland \\ E-mail:jagszcz@zut.edu.pl
}

\begin{abstract}
The three component reactions of Meldrum's acid, 1,3-bis-(benzotriazol-1-yl)propan-2-one or 1-benzotriazol-1-ylpropan-2-one and an aldehyde, carried out in DMF at $60{ }^{\circ} \mathrm{C}$ gave rise to 2,4-bis-(benzotriazol-1-yl)cyclohexane-1,3-diones or 4-benzotriazol-1-ylcyclohexane-1,3-diones, respectively, in fairly high yields. Unlike similar reactions reported earlier with acetone, the reactions with 1,3-bis-(benzotriazol-1-yl)propan-2-one and 1-benzotriazol-1-ylpropan-2-one proceeded via an intramolecular acylation of the other methyl group of acetone.
\end{abstract}

Keywords: 1,3-Bis-(benzotriazol-1-yl)propan-2-one, 1-benzotriazol-1-ylpropan-2-one, 2,4-bisbenzotriazol-1-yl-cyclohexane-1,3-diones, 2,2-dimethyl-1,3-dioxane-4,6-dione, Meldrum's acid

\section{Introduction}

Domino reactions ${ }^{1-3}$ are considered to be versatile synthetic tools and are used for the synthesis of structurally diverse compounds including biologically active natural products and drugs. ${ }^{2-5}$ As a strong CH-acid, Meldrum's acid readily enters into condensation reactions of the aldol ${ }^{6}$ and Knoevenagel $^{3,7}$ type to yield 5-alkenyl-2,2-dimethyl-1,3-dioxane-4,6-diones which are widely used in organic synthesis. With a vast number of diverse synthetic applications Meldrum's acid may be considered a unique synthon. Its methylidene derivatives, for instance, often are engaged as intermediates in multicomponent reactions (MCR) in which the Meldrum's acid moiety decomposes $^{2,3,12-14}$ as it is the case with intra- and intermolecular Friedel-Crafts $C$-alkylations ${ }^{15}$ and $\mathrm{NH}$-acylations of thioamides. ${ }^{16-18}$

\section{Results and Discussion}

The three component proline-catalyzed reaction of acetone with aldehydes and Meldrum's acid has been reported to yield triketones with no change in the 2,2-dimethyl-1,3-dioxane-4,6-dione (Meldrum's acid) ring. ${ }^{19}$ Analogous products have been noted in the piperidine-catalyzed 
reaction starting with cyclohexanone. ${ }^{20}$ Those results prompted us to investigate the reactions under conditions that could be favorable for inducing an intramolecular $C$-acylation of the other methyl group of benzotriazolo-acetone 1, 2. However, one could not rule out that the reaction may take another course and yield the enol form of the ketone $\mathbf{5}$ which subsequently may cyclize to give the corresponding lactone 14 .

The readily available acetone derivatives, namely 1,3-bis-benzotriazol-1-ylpropan-2-one $\mathbf{1}^{21}$ and 1-benzotriazol-1-ylpropan-2-one $\mathbf{2}^{22}$ were used in the present research as the active methylene compounds. All reactions were carried out at $60{ }^{\circ} \mathrm{C}$ in a DMF solution under inert gas with triethylamine added as the catalyst. As expected, methylidene derivatives $\mathbf{4}$ were formed in the first stage. Their subsequent Michael reaction with the ketone $\mathbf{1}$ or $\mathbf{2}$ anion yielded the addition product $\mathbf{5}$ which next underwent an intramolecular acylation with a concomitant decomposition of the Meldrum's acid fragment. An additional support to that mechanistic concept was gained in the reaction of $4\left(\mathrm{R}^{3}=\mathrm{Ph}\right)$ with 1 ; under identical reaction conditions (DMF, TEA, $60^{\circ} \mathrm{C}$ ) only 6 was formed, its yield being twice that high as in the three component variant (60\% vs. 30\%). The formation of lactones 14 or keto- esters 5 was not observed in our

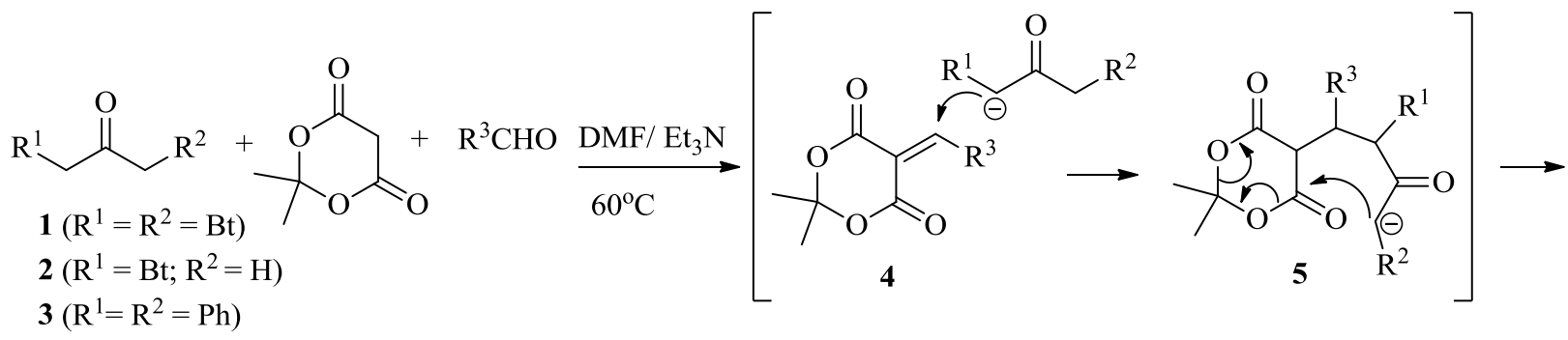

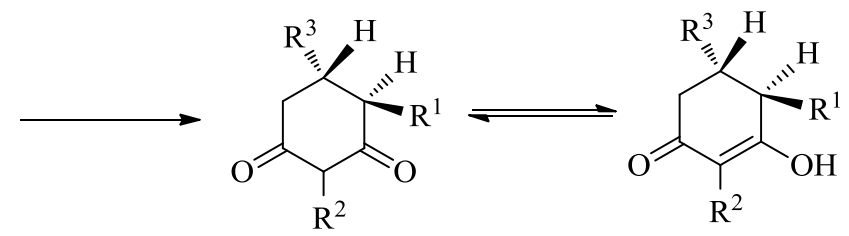

6 - 13<smiles>Cn1nnc2ccc(Br)cc21</smiles><smiles>[R]CC1=C([R])C([R])CC(=O)O1</smiles>

14

\begin{tabular}{cccccccccc}
\hline Cpd & $\mathrm{R}^{1}$ & $\mathrm{R}^{2}$ & $\mathrm{R}^{3}$ & $\begin{array}{c}\text { Yield } \\
(\%)\end{array}$ & $\mathrm{Cpd}$ & $\mathrm{R}^{1}$ & $\mathrm{R}^{2}$ & $\mathrm{R}^{3}$ & $\begin{array}{c}\text { Yield } \\
(\%)\end{array}$ \\
\hline $\mathbf{6}$ & $\mathrm{Bt}$ & $\mathrm{Bt}$ & $\mathrm{Ph}$ & $30(60)$ & $\mathbf{1 0}$ & $\mathrm{Bt}$ & $\mathrm{H}$ & $3,4-\left(\mathrm{OCH}_{2} \mathrm{O}\right) \mathrm{C}_{6} \mathrm{H}_{3}$ & 92 \\
$\mathbf{7}$ & $\mathrm{Bt}$ & $\mathrm{Bt}$ & $4-\mathrm{CH}_{3} \mathrm{C}_{6} \mathrm{H}_{4}$ & 52 & $\mathbf{1 1}$ & $\mathrm{Bt}$ & $\mathrm{H}$ & $\mathrm{Ph}$ & 51 \\
$\mathbf{8}$ & $\mathrm{Bt}$ & $\mathrm{Bt}$ & $\mathrm{CH}_{3}$ & 86 & $\mathbf{1 2}$ & $\mathrm{Bt}$ & $\mathrm{H}$ & $\mathrm{CH}_{3}$ & 81 \\
$\mathbf{9}$ & $\mathrm{Bt}$ & $\mathrm{Bt}$ & $3,4-\left(\mathrm{OCH}_{2} \mathrm{O}\right) \mathrm{C}_{6} \mathrm{H}_{3}$ & 85 & $\mathbf{1 3}$ & $\mathrm{H}$ & $\mathrm{H}$ & $3,4-\left(\mathrm{OCH}_{2} \mathrm{O}\right) \mathrm{C}_{6} \mathrm{H}_{3}$ & 10 \\
\hline
\end{tabular}

Scheme 1. Formation of cyclohexanediones. 
experiments. Since Knoevenagel condensation of aldehydes with benzotriazolo-acetone $\left(\mathbf{2}, \mathrm{R}^{1}=\right.$ $\mathrm{Bt}, \mathrm{R}^{2}=\mathrm{H}$ ) is known to require forced conditions (boiling DMF), ${ }^{23}$ the initial formation of the methylidene derivatives of $\mathbf{4}$ and their subsequent addition to Meldrum's acid seems rather unlikely.

It is noteworthy that the benzotriazole substituent were markedly effective when the acetone derivatives $\mathbf{1}$ and $\mathbf{2}$ formed the corresponding carbanions. ${ }^{24,25}$ Under analogous conditions the reaction of Meldrum's acid with benzaldehyde and 1,3-diphenylacetone 3 yielded a 1.5:1 mixture of the product of $\mathbf{3}$ addition to the appropriate methylidene derivative $\mathbf{4}$ and triphenyl1,3-cyclohexanedione 13; the reaction rate was much lower and the yield did not exceed $25 \%$. The ${ }^{1} \mathrm{H}-$ and ${ }^{13} \mathrm{C}$-NMR spectra confirmed the initial structural assignments and showed that in solutions the compounds 6-13 exist in the enol form.

The configurations at $\mathrm{C}-4$ (substituent $\mathrm{R}^{1}$ ) and at C-5 (substituent $\mathrm{R}^{3}$ ) were established by analysis of the vicinal coupling constants $\left({ }^{1} \mathrm{H},{ }^{1} \mathrm{H}\right.$ COSY NMR spectra) and of the through-space interactions $\left({ }^{1} \mathrm{H},{ }^{1} \mathrm{H}\right.$ NOESY NMR spectra) of compounds 8-10. High values $(8.2-10.5 \mathrm{~Hz})$ of the coupling constants between the vicinal protons $\mathrm{H}-4$ and $\mathrm{H}-5$ indicated their trans-diaxial orientation.

\section{Experimental Section}

General. Melting points were determined on a digital apparatus Electrothermal model IA9300 and are uncorrected. NMR spectra were recorded on a Bruker DPX apparatus (400 MHz) spectrometer in deuteriochloroform and deuteriodimethyl sulfoxide with tetramethylsilane as internal standard. In some cases trifluoroacetic acid (TFFA) was added to the solvent. The IR spectra were taken with Specord M80 instruments in potassium bromide pellets or Nujol. Purity and molecular mass determinations were carried out by gas chromatography-mass spectrometry (GC/MS) on a Hewlett-Packard instrument model HP 6890 equipped with a mass detector HP 5973. The analytical procedure was developed for a $30 \mathrm{~m}$ long capillary column, $0.2 \mathrm{~mm}$ in diameter, with methylsiloxane modified with phenyl groups (5\% $\mathrm{Ph}, \mathrm{Me}$ siloxane) in the 0.25 $\mu \mathrm{m}$ thick active phase layer. Elemental analyses were performed on EuroEA 3000 series, Euro Vector CHNS-O Elemental Analyzer.

\section{General procedure}

A mixture of dibenzotriazolacetone $\mathrm{e}^{21}$ or benzotriazolacetone ${ }^{22}(1 \mathrm{mmol})$, Meldrum's acid $(0,144 \mathrm{~g}, 1 \mathrm{mmol})$, appropriate aldehyde $(1 \mathrm{mmol})$ and triethylamine $(1 \mathrm{~mL})$ in anhydrous DMF $(5 \mathrm{~mL})$ was stirred and heated under argon blanket at $60{ }^{\circ} \mathrm{C}$ for a few hours. The reaction progress was controlled by thin-layer chromatography (silica gel, hexane/ethyl acetate, 1:1). After cooling $10 \mathrm{~mL}$ of $10 \%$ aq. $\mathrm{HCl}$ was added to the reaction mixture. The semisolid residue was filtered, dissolved in ethyl acetate, and chromatographed on $\mathrm{SiO}_{2}$ with hexane/ethyl acetate, 
1:1. The filtrate was evaporated to dryness and the product left was recrystallized from toluene/ hexane 6:4.

2,4-Bis-(1H-1,2,3-benzotriazol-1-yl)-5-phenylcyclohexane-1,3-dione (6). Light yellow oil, yield: $60 \%$, IR: $1695,1710 \mathrm{~cm}^{-1} .{ }^{1} \mathrm{H}$ NMR (400 MHz, DMSO- $\left.d_{6}\right): \delta 3.76-3.86\left(\mathrm{~m}, 2 \mathrm{H}, \mathrm{C}-\mathrm{CH}_{2}\right)$, 4.11-4.33 (m, CH-Ph), 5.73 (s, CH-Bt), 5.91-6.01 (m, $\left.\mathrm{OCH}_{2}\right), 7.11$ (d, J = 7.52 Hz, 2H, Ph), 7.21-7.30 (m, 2H, Bt), 7.32-7.44 (m, 5H, Bt, Ph), 7.93-8.11 (m, 4H, Bt), $11.62(\mathrm{~s}, 1 \mathrm{H}, \mathrm{OH}) .{ }^{13} \mathrm{C}$ NMR (100 MHz, DMSO-d $\left.)_{6}\right): \delta 36,91$ (d, $\left.\underline{\mathrm{C}} \mathrm{H}-\mathrm{Ph}\right), 53.42\left(\mathrm{t}, \mathrm{CH}_{2}\right), 69,48$ (d, $\left.\underline{\mathrm{C}}-\mathrm{Bt}\right), 125.19$, 128.61, 132.18, $142.79(\mathrm{Ph}), 110.64,113.72,119.60,122.55,127.02,127.33,129.06,130.17$, 136.11, 140.82, 145.45, 146.86 (Bt), 172.15 (s, =C-OH), 196.34 (s, C=O), MS: m/z: 422 (9), 351 (8), 281 (16), 260 (11), 220 (100), 207 (24), 117 (32), 77 (39). Anal. Calcd. for $\mathrm{C}_{24} \mathrm{H}_{18} \mathrm{~N}_{6} \mathrm{O}_{2}$ : C, 68.24; H, 4.29; N, 19.89. Found: C, 68.07; H, 4.28; N, $19.93 \%$.

2,4-Bis-(1H-1,2,3-benzotriazol-1-yl)-5-(4-methylphenyl)cyclohexane-1,3-dione (7). Dark yellow oil, yield: 52\%, IR: 1697, $1711 \mathrm{~cm}^{-1}$. ${ }^{1} \mathrm{H}$ NMR (400 MHz, DMSO-d $)$ ): $\delta 2.09$ (s, 3H, $\mathrm{CH}_{3}$ ), 3.86-3.99 (m, 2H, C-CH$)$, 4.16-4.26 (m, CH-Ph), 5.59 (s, CH-Bt), 7.15 (d, J = 7.66 Hz, 2H, Ph), 7.19-7.23 (m, 2H, Bt), 7.24-7.42 (m, 4H, Bt, Ph), 7.91-8.08 (m, 4H, Bt), 11.75 (s, 1H, $\mathrm{OH}) .{ }^{13} \mathrm{C}$ NMR (100 MHz, DMSO- $\left.d_{6}\right): \delta 21.11\left(\mathrm{q}, \mathrm{CH}_{3}\right), 42.39(\mathrm{~d}, \underline{\mathrm{CH}}-\mathrm{Ph}), 45.89\left(\mathrm{t}, \mathrm{CH}_{2}\right), 67.45$ (d, $\underline{\mathrm{CH}}-\mathrm{Bt}), 124.47,131.11,135.95,137.18(\mathrm{Ph}), 110.64,114.58,119.59,122.25,127.83$, 128.45, 128.86, 129.64, 135.75, 140.39, 145.40, $147.11(\mathrm{Bt}), 172.33$ (s, =C-OH), 197.25 (s, $\mathrm{C}=\mathrm{O}$ ), MS: m/z 436 (14), 351 (7), 281 (8), 260 (8), 220 (100), 207 (28), 117 (30), 77 (35). Anal. Calcd. for $\mathrm{C}_{25} \mathrm{H}_{20} \mathrm{~N}_{6} \mathrm{O}_{2}$ : C, 68.80; H, 4.62; N, 19.25. Found: C, 68.91; H, 4.60; N, 19.29\%.

2,4-Bis-(1H-1,2,3-benzotriazol-1-yl)-5-methylcyclohexane-1,3-dione (8). Light brown solid, yield: $86 \%$, mp. $148-150{ }^{\circ} \mathrm{C}$, IR: $1694,1706 \mathrm{~cm}^{-1} .{ }^{1} \mathrm{H}$ NMR (400 MHz, DMSO-d $\left.d_{6}\right): \delta 1.67(\mathrm{~s}$,

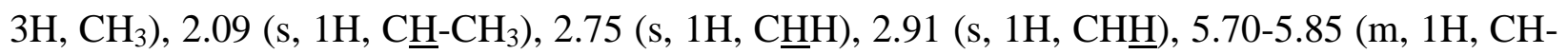
$\mathrm{Bt}), 7.41$ (t, $J=6.94 \mathrm{~Hz}, 2 \mathrm{H}, \mathrm{Bt}), 7.57$ (t, $J=7.90 \mathrm{~Hz}, 2 \mathrm{H}, \mathrm{Bt}), 7.88$ (d, $J=7.94 \mathrm{~Hz}, 2 \mathrm{H}, \mathrm{Bt})$, 7.99-8.11 (m, 2H, Bt), $11.65(\mathrm{~s}, 1 \mathrm{H}, \mathrm{OH}) .{ }^{13} \mathrm{C}$ NMR (100 MHz, DMSO-d $\left.)_{6}\right) \delta 17.39\left(\mathrm{q}, \mathrm{CH}_{3}\right)$, 34.74 (d, $\left.\underline{\mathrm{CH}}-\mathrm{CH}_{3}\right), 45.87$ (t, $\left.\mathrm{CH}_{2}\right), 67.43$ (d, CH-Bt), 111.26, 114.06, 119.54, 122.36, 127.90, 128.72, 128.83, 129.63, 134.11, 140.51, 145.46, 147.19 (Bt), 173.41 (s, C-OH), 197.22 (s, C=O), MS: m/z 360 (16), 261 (10), 207 (8), 184 (19), 166 (15), 144 (99), 123 (17), 104 (30), 77 (100), 51 (19). Anal. Calcd. for $\mathrm{C}_{19} \mathrm{H}_{16} \mathrm{~N}_{6} \mathrm{O}_{2}$ : C, 63.32; H, 4.48; N, 23.32. Found: C, 63.26; H, 4.47; N, $23.36 \%$.

5-(1,3-Benzodioxol-5-yl)-2,4-bis-(1H-1,2,3-benzotriazol-1-yl)cyclohexane-1,3-dione

(9). Yellow solid, yield: 85\%, mp. 167-170 ${ }^{\circ} \mathrm{C}$, IR: 1690, $1715 \mathrm{~cm}^{-1} .{ }^{1} \mathrm{H}$ NMR (400 MHz, DMSO$\left.d_{6}\right): \delta$ 2.51-2.62 (m, 2H, C-CH$), 3.24-3.44\left(\mathrm{~m}, 1 \mathrm{H}, \mathrm{C} \underline{\mathrm{H}}-3,4-\left(\mathrm{OCH}_{2} \mathrm{O}\right) \mathrm{C}_{6} \mathrm{H}_{3}, 6.16\left(\mathrm{~s}, 2 \mathrm{H}, \mathrm{OCH}_{2}\right)\right.$, $6.98\left(\mathrm{~d}, J=7.81 \mathrm{~Hz}, 2 \mathrm{H}, 3,4-\left(\mathrm{OCH}_{2} \mathrm{O}\right) \mathrm{C}_{6} \underline{\mathrm{H}}_{3}\right), 7.38(\mathrm{t}, J=6.81 \mathrm{~Hz}, 1 \mathrm{H}, \mathrm{Bt}), 7.51$ (t, $J=7.37 \mathrm{~Hz}$, $1 \mathrm{H}, \mathrm{Bt}), 7.61(\mathrm{~d}, J=7.81 \mathrm{~Hz}, 2 \mathrm{H}, \mathrm{Bt}), 7.78\left(\mathrm{~d}, J=7.36 \mathrm{~Hz}, 1 \mathrm{H}, 3,4-\left(\mathrm{OCH}_{2} \mathrm{O}\right) \mathrm{C}_{6} \underline{\mathrm{H}}_{3}\right), 7.95-8.06$ $(\mathrm{m}, 4 \mathrm{H}, \mathrm{Bt}), 11.26(\mathrm{~s}, 2 \mathrm{H}, 2 \mathrm{OH}) .{ }^{13} \mathrm{C} \mathrm{NMR}\left(100 \mathrm{MHz}, \mathrm{DMSO}-d_{6}\right): \delta 30.98(\mathrm{~d}, \mathrm{CH}-3,4-$ $\left.\left(\mathrm{OCH}_{2} \mathrm{O}\right) \mathrm{C}_{6} \mathrm{H}_{3}\right), 54.33\left(\mathrm{t}, \mathrm{C}-\underline{\mathrm{CH}}_{2}\right), 102.40\left(\mathrm{t}, \mathrm{OCH}_{2}\right), 103.89$ (s, =C-Bt), 108.34 (s, =C-Bt), $110.44,111.45,111.69,119.13,123.81,125.99,127.31,133.69,134.05,145.24,147.86,152.84$, $\left.157.00\left(\mathrm{Bt}, 3,4-\left(\mathrm{OCH}_{2} \mathrm{O}\right) \underline{\mathrm{C}}_{6} \mathrm{H}_{3}\right)\right), 159.91$ (s, =C-OH), 163.10 (s, =C-OH), MS: decomposition. Anal. Calcd. for $\mathrm{C}_{25} \mathrm{H}_{18} \mathrm{~N}_{6} \mathrm{O}_{4}$ : C, 64.37; H, 3.89; N, 18.02. Found: C, 64.52; H, 3.88; N, $18.05 \%$. 
5-(1,3-Benzodioxol-5-yl)-4-(1H-1,2,3-benzotriazol-1-yl)cyclohexane-1,3-dione (10). Brown oil, yield: 92\%, IR: $1695,1712 \mathrm{~cm}^{-1} .{ }^{1} \mathrm{H}$ NMR (400 MHz, $\left.\mathrm{CDCl}_{3}\right): \delta 3.95-4.10\left(\mathrm{~m}, 2 \mathrm{H}, \mathrm{C}_{-} \mathrm{CH}_{2}\right.$ ), 4.26-4.42 (m, 1H, Cㅍ-3,4- $\left.\left(\mathrm{OCH}_{2} \mathrm{O}\right) \mathrm{C}_{6} \mathrm{H}_{3}\right), 5.68$ (s, 1H, CH-Bt), 5.80-5.93 (m, 2H, $\left.\mathrm{OCH}_{2}\right), 6.46$ $(\mathrm{s}, 1 \mathrm{H},=\underline{\mathrm{C}} \mathrm{H}-\mathrm{C}=\mathrm{O}), 6.70\left(\mathrm{t}, J=9.72 \mathrm{~Hz}, 2 \mathrm{H}, 3,4-\left(\mathrm{OCH}_{2} \mathrm{O}\right) \mathrm{C}_{6} \underline{\mathrm{H}}_{3}\right), 7.25(\mathrm{~s}, 1 \mathrm{H}, \mathrm{Bt}), 7.29-7.40$ (m, $2 \mathrm{H}, \mathrm{Bt}), 7.47$ (t, $\left.J=7.67 \mathrm{~Hz}, 1 \mathrm{H}, 3,4-\left(\mathrm{OCH}_{2} \mathrm{O}\right) \mathrm{C}_{6} \underline{\mathrm{H}}_{3}\right), 7.96-8.04$ (m, 1H, Bt), 11.84 (br. s., 1H, $\mathrm{OH}) .{ }^{13} \mathrm{C} \mathrm{NMR}\left(100 \mathrm{MHz}, \mathrm{CDCl}_{3}\right): \delta 28.50\left(\mathrm{~d}, \underline{\mathrm{CH}}-3,4-\left(\mathrm{OCH}_{2} \mathrm{O}\right) \mathrm{C}_{6} \mathrm{H}_{3}\right), 60.40\left(\mathrm{t}, \mathrm{C}-\mathrm{CH}_{2}\right), 70.59$ (d, CH-Bt), 100.85 (t, $\mathrm{OCH}_{2}$ ), 107.99 (d, O=C-CH=), 109.75, 110.31, 120.00, 121.51, 124.45, 124.64, 127.9, 132.48, 145.21, 154.53, 158.40 (Bt, 3,4-( $\left.\left.\mathrm{OCH}_{2} \mathrm{O}\right) \underline{\mathrm{C}}_{6} \mathrm{H}_{3}\right), 171.24$ (s, C-OH), 190.43 (s, C=O), MS: m/z 349 (17), 293 (24), 274 (19), 250 (100), 220 (25), 192 (11), 147 (66), 89 (21), 77 (12), 63 (9), 43 (31). Anal. Calcd. for $\mathrm{C}_{19} \mathrm{H}_{15} \mathrm{~N}_{3} \mathrm{O}_{4}$ : C, 65.32; H, 4.33; N, 12.03. Found: C, 65.26; H, 4.35; N, 12.06\%.

4-(1H-1,2,3-Benzotriazol-1-yl)-5-phenylcyclohexane-1,3-dione (11). Red oil, yield: 51\%, IR: 1692, $1715 \mathrm{~cm}^{-1} .{ }^{1} \mathrm{H}$ NMR $\left(400 \mathrm{MHz}, \mathrm{CDCl}_{3}\right): \delta 3.86-3.99\left(\mathrm{~m}, 2 \mathrm{H}, \mathrm{C}-\mathrm{CH}_{2}\right), 4.16-4.35(\mathrm{~m}, 1 \mathrm{H}$, CH-HT), 5.87 (s, 1H, CH-Bt), 6.46 (s, 1H, =CH-C=O), 7.25 (s, 2H, Ph), 7.31-7.52 (m, 6H, Bt, $\mathrm{Ph}), 8.06$ (d, $J=8.65 \mathrm{~Hz}, 1 \mathrm{H}, \mathrm{Bt}), 11.66$ (br. s., $1 \mathrm{H}, \mathrm{OH}) .{ }^{13} \mathrm{C} \mathrm{NMR}\left(100 \mathrm{MHz}, \mathrm{CDCl}_{3}\right): \delta 31.94$ (d, CH-Ph), 60.95 (t, C-CH ), 71.51 (d, CH-Bt), 100.68 (d, O=C-CH=), 110.50, 118.52, 124.56, 125.72, 126.31, 131.27, 133.86, 142.72, 145.66 (Bt, Ph), 172.34 (s, C-OH), 195.43 (s, C=O), MS: $m / z$ 305(2), 263(8,7), 235(14,5), 206(71), 131(100), 103(61), 77(85), 51(23). Anal. Calcd. for $\mathrm{C}_{18} \mathrm{H}_{15} \mathrm{~N}_{3} \mathrm{O}_{2}: \mathrm{C}, 70.81 ; \mathrm{H}, 4.95 ; \mathrm{N}, 13.76$. Found: 70.71; H, 4.95; N, $13.72 \%$.

4-(1H-1,2,3-Benzotriazol-1-yl)-5-methylcyclohexane-1,3-dione (12). Light brown solid, yield: 81\%, IR: 1695, $1725 \mathrm{~cm}^{-1} .{ }^{1} \mathrm{H}$ NMR (400 MHz, DMSO- $\left.d_{6}\right): \delta 1.75\left(\mathrm{~s}, 3 \mathrm{H}, \mathrm{CH}_{3}\right), 2.07(\mathrm{~s}, 1 \mathrm{H}$,

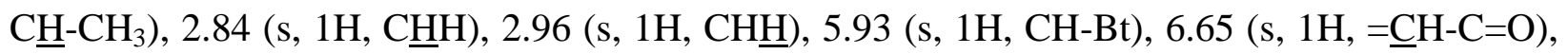
$7.50(\mathrm{~d}, J=5.66 \mathrm{~Hz}, 1 \mathrm{H}, \mathrm{Bt}), 7.58-7.78(\mathrm{~m}, 1 \mathrm{H}, \mathrm{Bt}), 7.81(\mathrm{~d}, J=7.37 \mathrm{~Hz}, 1 \mathrm{H}, \mathrm{Bt}), 8.13(\mathrm{~d}, J=$ $6.94 \mathrm{~Hz}, 1 \mathrm{H}, \mathrm{Bt}), 11.57$ (br. s, $1 \mathrm{H}, \mathrm{OH}) .{ }^{13} \mathrm{C}$ NMR (DMSO-d $): \delta 14.62\left(\mathrm{q}, \mathrm{CH}_{3}\right), 31.13(\mathrm{~d}, \underline{\mathrm{CH}}-$ $\left.\mathrm{CH}_{3}\right), 56.86$ (t, $\left.\mathrm{CH}_{2}\right), 69.41$ (d, CH-Bt), 106.01 (d, $\left.\mathrm{O}=\mathrm{C}-\mathrm{CH}=\right), 111.20,119.46,124.39,127.79$, 134.01, 145.79 (Bt), $166.23(\mathrm{C}-\mathrm{OH}), 201.62$ (s, C=O), MS: m/z 243 (5), 200 (10), 186 (6), 172 (43), 158 (9), 144 (100), 130 (17), 124 (10), 117 (10), 104 (19), 91 (18), 77 (31), 50 (27), 43 (77), 29 (8). Anal. Calcd. for $\mathrm{C}_{13} \mathrm{H}_{13} \mathrm{~N}_{3} \mathrm{O}_{2}$ : C, 64.19; H, 5.39; N, 17.27. Found: C, 64.31; H, 5.36; $\mathrm{N}, 17.30 \%$.

5-(1,3-Benzodioxol-5-yl)-2,4-diphenylcyclohexane-1,3-dione (13). Yellow solid, yield: 10\%, mp 181-183 ${ }^{\circ} \mathrm{C}$, IR: 1690 (C=O). ${ }^{1} \mathrm{H}$ NMR (400 MHz, DMSO-d $\left.d_{6}\right): \delta 2.56-2.72\left(\mathrm{~m}, 2 \mathrm{H}, \mathrm{C}-\mathrm{CH}_{2}\right)$, 3.69-3.79 (m, 1H, Cㅍ-3,4- $\left.\left(\mathrm{OCH}_{2} \mathrm{O}\right) \mathrm{C}_{6} \mathrm{H}_{3}\right), 3.98$ (d, J = 6.54 Hz, 1H, CH-Ph), 4.30 (s, 1H, CH$\mathrm{Ph}), 5.99\left(\mathrm{~s}, 2 \mathrm{H}, \mathrm{OCH}_{2}\right), 6.92\left(\mathrm{~d}, J=7.75 \mathrm{~Hz}, 2 \mathrm{H}, 3,4-\left(\mathrm{OCH}_{2} \mathrm{O}\right) \mathrm{C}_{6} \underline{\mathrm{H}}_{3}\right), 6.99-7.08$ (m, 1H, 3,4$\left.\left(\mathrm{OCH}_{2} \mathrm{O}\right) \mathrm{C}_{6} \underline{\mathrm{H}}_{3}\right), 7.11-7.24(\mathrm{~m}, 4 \mathrm{H}, \mathrm{Ph}), 7.25-7.32(\mathrm{~m}, 4 \mathrm{H}, \mathrm{Ph}), 7.35-7.42(\mathrm{~m}, 2 \mathrm{H}, \mathrm{Ph}) .{ }^{13} \mathrm{C} \mathrm{NMR}$ (100 MHz, DMSO- $\left.d_{6}\right): \delta 30.23\left(\mathrm{~d}, \underline{\mathrm{CH}}-3,4-\left(\mathrm{OCH}_{2} \mathrm{O}\right) \mathrm{C}_{6} \mathrm{H}_{3}\right), 45.45\left(\mathrm{t}, \mathrm{C}-\mathrm{CH}_{2}\right), 54.63(\mathrm{~d}, \mathrm{CH}-\mathrm{Ph})$, 68.46 (d, CH-Ph), 101.53 (t, $\left.\mathrm{OCH}_{2}\right), 108.98,110.73,121.53,126.17,127.93,127.97,128.04$, $128.15,130.48,135.40,137.35,139.63,146.03,148.88\left(2 \mathrm{Ph}, 3,4-\left(\mathrm{OCH}_{2} \mathrm{O}\right) \underline{\mathrm{C}}_{6} \mathrm{H}_{3}\right), 203.11$ (s, $\mathrm{C}=\mathrm{O}$ ), 205.29 (s, C=O), MS: m/z 384 (3), 320 (11), 292 (8), 274 (11), 216 (26), 188 (37), 131 (9), 104 (100), 42 (9). Anal. Calcd. for $\mathrm{C}_{25} \mathrm{H}_{20} \mathrm{O}_{4}$ : C, 78.11; H, 5.24. Found: C, 77.99; H, 5.23\%. 
3-(1,3-Benzodioxol-5-yl)-5-hydroxy-4,6-diphenylcyclohexa-1,5-dien-1-one (5). Orange oil, yield: $15 \%$, IR $1685(\mathrm{C}=\mathrm{O}) .{ }^{1} \mathrm{H}$ NMR (400 MHz, DMSO- $\left.d_{6}\right): \delta 2.75(\mathrm{~d}, J=8.65 \mathrm{~Hz}, 1 \mathrm{H}$, $\mathrm{CH}=\mathrm{C}=\mathrm{O}), 3.38-3.49\left(\mathrm{~m}, 1 \mathrm{H}, \mathrm{C} \underline{\mathrm{H}}-3,4-\left(\mathrm{OCH}_{2} \mathrm{O}\right) \mathrm{C}_{6} \mathrm{H}_{3}\right), 4.36(\mathrm{~d}, J=7.74 \mathrm{~Hz}, 1 \mathrm{H}, \mathrm{CH}-\mathrm{Ph}), 6.10(\mathrm{~s}$, $=\mathrm{CH}-\mathrm{Ph}), 6.00\left(\mathrm{~s}, 2 \mathrm{H}, \mathrm{OCH}_{2}\right), 6.81-6.92\left(\mathrm{~m}, 3 \mathrm{H}, 3,4-\left(\mathrm{OCH}_{2} \mathrm{O}\right) \mathrm{C}_{6} \underline{\mathrm{H}}_{3}\right), 7.19-7.34(\mathrm{~m}, 8 \mathrm{H}, \mathrm{Ph})$, 7.59-7.65 (m, 2H, Ph), $13.89(\mathrm{~s}, 1 \mathrm{H}, \mathrm{C}-\mathrm{OH}) .{ }^{13} \mathrm{C}$ NMR (100 MHz, DMSO-d $\left.)_{6}\right): \delta 0.72(\mathrm{~s}$, $\underline{\mathrm{C}}=\mathrm{C}=\mathrm{C}), 41.26\left(\mathrm{~d}, \underline{\mathrm{CH}}-3,4-\left(\mathrm{OCH}_{2} \mathrm{O}\right) \mathrm{C}_{6} \mathrm{H}_{3}\right), 53.97(\mathrm{~d}, \mathrm{CH}-\mathrm{Ph}), 101.10\left(\mathrm{t}, \mathrm{OCH}_{2}\right), 106.03(=\mathrm{CH}-$ $\mathrm{Ph}), 109.50,110.13,123.16,125.64,126.65,128.31,128.34,128.35,128.56,134.68,135.70$, 142.54, 146.12, 149.65 (2Ph, 3,4- $\left.\left(\mathrm{OCH}_{2} \mathrm{O}\right) \underline{\mathrm{C}}_{6} \mathrm{H}_{3}\right) .170 .09$ (s, C-OH), 197.81 (s, C=O), MS: decomposition, Anal. Calcd. for $\mathrm{C}_{25} \mathrm{H}_{20} \mathrm{O}_{4}$ : C, 78.11; H, 5.24. Found: C, 78.06; H, 5.26\%.

\section{Acknowledgements}

We express our thanks to Professor Jerzy Lange of the Warsaw University of Technology for helpful discussions and assistance with the manuscript.

\section{References}

1. Ho, T. L. Tandem Organic Reactions, Wiley: New York, 1992.

2. Tietze, L. F. Pure Appl. Chem. 2004, 76, 1967.

3. Ivanov A. S. Chem. Soc. Rev. 2008, 37, 789 and references cited therein.

4. Tietze, L. F.; Modi, A. Med. Res. Rev. 2000, 20, 304.

5. Tietze, L. F. Chem. Rev. 1996, 96,115 and references cited therein.

6. Zhu, A.; Jiang, T.; Wang, D.; Han, B.; Liu, L.; Huang, J.; Zhang, J.; Sun, D. Green Chem. 2005, 7, 514.

7. Dumas, A. M.; Seed, A.; Zorzitto, A.K.; Fillon, E. Tetrahedron Lett. 2007, 48, 7072.

8. Knoepfel, T. F.; Zarotti, P.; Ichikawa, T.; Carreira, E. M. J. Am. Chem. Soc. 2005, 127, 9682.

9. Watanabe, T.; Knoepfel, T. F.; Carreira, E. M. Org. Lett. 2003, 5, 4557.

10. Filion, E.; Dumas. A. M.; Hogg. S. A. J. Org. Chem. 2006, 71, 9899 and references cited therein.

11. Ramachary, D. B.; Chowdara, N. S.; Barbas, C. F. Angew. Chem. Int. Ed. 2003, 42, 42333.

12. Gaber, A. E.-A. O.; McNab, H. Synthesis 2001, 2059.

13. Chen, B. C. Heterocycles 1991, 32, 529.

14. Lipson, V. V.; Gorobets, N. Y. Mol. Divers. 2009, 13, 339.

15. Dumas, A. M.; Fillion, E. Accounts Chem. Res. 2010, 43, 440 and references cited therein.

16. Jagodziński, T. S. Chem. Rev. 2003, 103, 197 and references cited therein.

17. Li-Rong Wen, Chen Ji, Ming Li, Huai-Yuan Xie Tetrahedron 2009, 65, 1287.

18. Frolov, K. A.; Dotsenko, V. V.; Krivokolysko, S. G.; Litvinov, V. P. Russ. Chem. Bull. Int. Ed. 2005, 54, 1335. 
19. List, B.; Castello, C. Synlett 2001, 1687.

20. Skuratova, M. I.; Fedotova, O. V.; Kharchenko, V. G. Russian J. Org. Chem. 2002, 38, 1380.

21. Katritzky, A. R.; Wu, J. Synthesis 1994, 597.

22. Pchelka, B. K.; Loupy, A.; Petit, A. Tetrahedron: Asymmetry 2006, 17, 2516.

23. Al-Omran, F.; Abou El-Khair, A. J. Heterocyclic Chem. 2004, 41, 327.

24. Katritzky, A. R.; Kirichenko, K. Arkivoc 2006, (iv), 119.

25. Katritzky, A. R.; Ji, Y., Tymoshenko, D. O.; Fang Y.; Hylton, K-G. Arkivoc 2001, (iv), 20. 